This is an openly available pre-print of the paper.

To access the published PDF in the journal Motivation and Emotion, go to: https://link.springer.com/article/10.1007/s11031-019-09768-8

\title{
Moral self-judgment is stronger for future than past actions
}

\author{
Hallgeir Sjåstad \\ Norwegian School of Economics and SNF - Centre for Applied Research at NHH
}

Roy F. Baumeister

University of Queensland and Florida State University

April, 2019

Word Count: 12,557

\section{Author contact:}

Correspondence concerning this article should be addressed to Hallgeir Sjåstad, FAIR Insight Team, Norwegian School of Economics and SNF - Centre for Applied Research at NHH. E-mail: $\underline{\text { Hallgeir.Sjastad@snf.no }}$

\section{Funding:}

This work was partly supported by the Norway-America Association (NORAM), and the Research Council of Norway through its Centres of Excellence Scheme, FAIR project No 262675. Data and materials for all four studies are openly available online: www.osf.io/z95rm. 


\begin{abstract}
When, if ever, would a person want to be held responsible for his or her choices? Across four studies $(N=915)$, people favored more extreme rewards and punishments for their future than their past actions. This included thinking that they should receive more blame and punishment for future misdeeds than for past ones, and more credit and reward for future good deeds than for past ones. The tendency to moralize the future more than the past was mediated by anticipating (one's own) emotional reactions and concern about one's reputation, which was stronger in the future as well. The findings fit the pragmatic view that people moralize the future partly to guide their choices and actions, such as by increasing their motivation to restrain selfish impulses and build long-term cooperative relationships with others. People typically believe that the future is open and changeable, while the past is not. We conclude that the psychology of moral accountability has a strong future component.
\end{abstract}

Word Count: 159

Keywords: morality; self-judgment; prospection; emotion; reputation 


\section{Introduction}

People make many choices that have moral implications, but the processes of assigning and embracing praise and blame for these choices are often less than rigorously consistent and evenhanded (e.g., Alicke, 2000). Accepting responsibility for one's own choices is especially important, but it is also risky, insofar as it may bring guilt and invite punishment. The present investigation explored a seemingly paradoxical aspect of accepting accountability for one's own choices, which is that people may assert their future misdeeds should be punished more severely than their past ones. The paradox lies in the notion that moral principles are widely regarded as timeless, so the wrongness of an immoral action should not depend on its timing, especially if the circumstances are the same. Caruso's (2010, p. 610) seminal investigation began by noting that "Logically, an unethical behavior performed yesterday should also be unethical if performed tomorrow"- but then went on to report six studies in which people judged identical transgressions (by other people) more harshly when described as occurring in the future than in the past. In addition, a seventh study showed that people rated a future donation as more generous than an identical donation in the past, even though inflation technically entails that the objective monetary value of the future donation would be less.

Why would future actions be subjected to more extreme moral judgment than past ones? Caruso (2010) explained his findings based on Frijda's (1986) theory that emotions function to prepare people for actions. Such an explanation was a reasonable first step, but we sought to build on the explanation by addressing some key questions. In particular, Frijda's analysis pertains to a person's own actions, but Caruso's studies all involved judging the actions of other people (or corporations or even machines). Our analysis therefore focused on the crucial question of whether people would also call for more extreme consequences for their own future than past actions. 
To be sure, the apparent moralization of the future may not be as moral as it appears. One could interpret Caruso's (2010) findings as indicating strictly selfish motivations. A person's future prospects are better if other people behave morally rather than immorally, and so even a purely selfish person may want others to be judged and punished by high standards. However, such an analysis would predict that people would stop far short of wanting higher or harsher standards for their own actions. Hence, much rides on whether Caruso's moralization effects extend to how people would judge their own future versus past actions.

Indeed, a large literature has indicated that people judge themselves differently from how they judge others. Ability attributions show primacy effects for others but recency effects for self (Jones, Rock, Shaver, Goethals, \& Ward, 1968). There is some tendency for people to attribute other people's actions to inner, dispositional causes but to attribute their own actions to external, situational causes (Jones \& Nisbett, 1971), which already suggests that they expect their own moral misdeeds to be judged and excused by a different set of standards than those that apply to other people. People construe their own behavior with self-serving attributional biases (Zuckerman, 1979) and other self-flattering patterns (for reviews, see Greenwald, 1980; Taylor \& Brown, 1988; Balcetis \& Cardenas, 2018) that do not enter into their judgments of others. People judge other people by their actions but themselves by their intentions (Kruger \& Dunning, 1999), which again suggests different criteria for moral evaluation. In view of these and other differences, it would be remarkable if the more stringent moral evaluation of other people's future than past actions were duplicated with one's own actions. Yet there are some reasons to hypothesize that this could happen, which we now elucidate. 


\section{Pragmatic Prospection and Moralizing the Future}

One reason for greater investment in thinking about the future than the past is that it is more useful for guiding one's choice of action. The past cannot be changed, but the future can. Although psychologists have studied the past (e.g., memory) much more than the future, people tend to think more about the future than the past (Seligman, Railton, Baumeister, \& Sripada, 2013). In addition, although most psychological research on the future has emphasized predicting what will happen, Baumeister, Vohs, and Oettingen (2016) have argued that future thinking is often primarily pragmatic, in the sense that people think about what they need to do in choice and performance situations to select a response from multiple possibilities. Planning is more frequent than predicting. If a major purpose of moral judgment is to inform and guide action, then morality is much more pragmatically relevant to future than past actions.

Caruso (2010) noted the relevance of prospective thinking for moral judgment of others. That applies to the self as well, however. People cannot undo past misdeeds, but they can prevent themselves from committing future ones. By inflating the appropriate level of punishment for future misdeeds, people may be striving to prevent themselves from committing such immoral actions in the future.

To be sure, one might ask from a self-interest perspective why a person should seek to behave virtuously in the future at all, let alone more than in the past. Evolutionary analyses suggest an answer. Humans evolved to cooperate with others, including non-kin, much more than other primates have (e.g., Suddendorf, 2013). Tomasello (2014) has pointed out that cooperation requires moral prospection much more than other forms of primate social behavior - because one must attract partners for future cooperation. That is, insofar as humans survive and reproduce by cooperating, a person's success and very life may depend on inducing others to cooperate. Cooperation breaks down when two or more individuals 
work together for some reward, but then one party takes it all without sharing. Someone who does that, however, will soon run out of willing partners for future cooperative ventures. That is, reputation is an important component of long-term cooperation strategies (Rand \& Nowak, 2013), and people are willing to make substantial sacrifices to protect their reputation from harm (Vonasch, Reynolds, Winegard, \& Baumeister, 2018). Hence, what enables the individual to resist the temptation to selfishly betray partners is the concern with his or her own future reputation (see also Ybarra, Park, Stanik, \& Lee, 2012; Pagliaro, Ellemers, Baretto, \& Di Cesare, 2016; Sjåstad, 2019). We turn now to our main hypotheses.

\section{Hypotheses}

\section{Future Moralization: Increased Rewards and Punishments}

The primary hypothesis was that people will moralize the future, in the sense that they would raise the moral stakes for their own future as compared to past actions. More precisely, we predicted that people would prescribe more blame and punishment for their future misdeeds than past ones, and more praise and reward for future good deeds than past ones. A future moralization effect of this kind would extend Caruso's (2010) findings with the important step of applying them to how people judge themselves. Beyond that, we articulated two explanatory hypotheses to explain why that pattern would occur. They are not mutually exclusive but are independent, so either, both, or neither could be correct.

\section{Reputational Concern}

Our first explanatory hypothesis was already stated, namely that the concern over one's moral reputation would drive the effects. That is, people will worry more about how others will evaluate them in the future, and so they will call for more severe rewards and punishments in order to motivate themselves to behave properly in the future. 


\section{Anticipated Emotion}

A second explanatory hypothesis was that emotion would be decisive. Caruso's (2010) work revealed that emotions play a key role in the moralization of other people's future actions. Presumably people's emotions are even more relevant to their own actions than to other people's actions, so if people do call for greater rewards and punishments for future than past actions, emotion could be a prominent and potent mediator.

The reason that emotion could contribute to temporal differences in moral judgment is not entirely clear, however. Caruso (2010) built his reasoning on Frijda's (1986) theory that the purpose of emotions is to prepare for action - but Caruso's participants were not preparing for action, and indeed it is unclear why preparing for their own actions would cause emotional reactions to future actions by others who are not relevant to the self. Moreover, Frijda's theory is somewhat at odds with the evidence (mostly accumulated subsequent to his theoretical statement) showing that emotion does not have strong or direct ties to behavior. Reviewing the extensive literature on emotion, Clore and Huntsinger (2007) noted that there were remarkably few findings linking emotion to behavior — in contrast to the extensive evidence linking emotion to cognitive processes, such as social judgment (see also Schwarz \& Clore, 2003). A recent meta-analysis by DeWall, Baumeister, Chester, and Bushman (2016) found that currently felt emotion was significantly related to behavioral outcomes in only a small minority of cases.

An alternative theory of how emotion is indirectly related to behavior, via influences on cognition and learning, was proposed by Baumeister, Vohs, DeWall, and Zhang (2007). They treated emotion as part of a feedback loop. Emotion involves evaluation of motivationally relevant outcomes, and it stimulates cognitive processing of information, including the processing of one's own recent actions and outcomes. Thus, it facilitates learning lessons for the future. Also, crucially, people gradually learn what courses of action 
will produce what emotional outcomes, and they adjust their behavior so as to do what will bring positive emotions and avoid negative ones.

Such a process is highly relevant to moral learning. People presumably learn morality when they first act in self-interested ways, which is the natural tendency, but which sometimes elicits disapproval, social exclusion, and even punishment from others. Anticipating objections to one's actions becomes internalized as guilt. Therefore, people learn to analyze their behavior from a moral perspective to learn what actions will lead to feeling guilty, and then they generally avoid those actions. Indeed, Baumeister et al. (2007) noted that guilt is a paradigmatic instance of the feedback theory of emotion, in contrast to fear being the favorite of the theory that emotion directly causes behavior. (In the standard example, fear conferred a natural selection advantage because it made people escape from danger, whereas hominids lacking fear were killed.) A recent meta-analysis by Leach and Cidam (2015) extended this approach by showing that shame and guilt mainly increase pro-social behavior insofar as the damage to one's reputation is reparable. Thus, the emotion in the form of feeling bad about a past misdeed functions to orient people to seek ways of rectifying the spoiled identity and achieving an improved reputation in the future.

A relevant aspect of emotion comes from research on affective forecasting (e.g., Wilson \& Gilbert, 2005). The main finding has been that people typically predict the correct emotion, but overestimate the intensity and duration of these emotions, good and bad. Caruso (2010) invoked that work to explain his findings. Oddly, though, his measures focused on present emotion rather than future or anticipated emotion, and thus did not involve affective forecasting. Possibly the two are correlated, and a prior study found that anticipating future experiences caused stronger emotion in the present than remembering similar experiences from the past (Van Boven \& Ashworth, 2007). Still, the current investigation overtly sought 
to measure anticipated emotion in connection with future moral and immoral actions, as compared to retrospective emotion about past actions.

The affective forecasting tendency to overestimate future emotions can be regarded as simply another cognitive deficit or judgment error typical of the flawed human mind. Alternatively, however, that tendency does fit very well with the feedback theory of emotion. As Baumeister et al. (2007) argued, if people guide their behavior on the basis of anticipated emotion, then it would be adaptive for the person to anticipate strong emotion because that is more motivating. For example, one would presumably try harder to avoid weeks of feeling intensely guilty than to avoid a fleeting twinge of guilt. After the fact, some of the expected emotion is necessary to sustain the feedback loop, but it does not have to last as long as anticipated — which is precisely the pattern usually found (Wilson \& Gilbert, 2005). The same should be the case for positive emotions in connection to moral actions: Overestimating how good it will feel to do the right thing may motivate the person to actually do it. Hence, our second explanatory hypothesis was that people would attribute more responsibility to themselves for future actions because they would also expect stronger emotional reactions in connection with future than past moral and immoral actions.

\section{Present Investigation Overview}

We report four experiments with a total of 915 participants, testing a series of hypotheses about how people make moral judgments and set appropriate levels of reward and punishment for themselves for future and past actions (see fig. 1). We conducted Study 1 with a university student sample in Norway. The relatively young average age raised some alternative explanations, so the other studies used online samples from the United States, which allowed us to avoid those problems. The central hypothesis was that people would deem appropriate rewards and punishments to be greater for their future than past actions, and 
that this would be true for both virtuous and immoral actions. To explore what mechanisms that could explain this effect, we also tested the two explanatory hypotheses concerning anticipated emotion and reputational concern. In addition, we explored moral optimism and (lack of) personal relevance as possible alternative explanations.

Relying on previous work on temporal asymmetries in human judgment (Rothbart \& Snyder, 1970; Caruso, Gilbert, \& Wilson, 2008; Caruso, 2010; Helzer \& Gilovich, 2012; Ferrante, Girotto, Stragà, \& Walsh, 2013), the methodological approach in the current investigation relies on framing manipulations. Across all our four studies, participants were asked to make moral judgments of their own actions that are described as occurring either in the past or in the equidistant future. In order to strengthen the manipulation, our initial instruction also gave participants a prompt to focus on the time frame they were assigned to in the following questions (past or future). In essence, this procedure enabled a systematic comparison of whether people tend to moralize their future actions more than similar actions from the past.

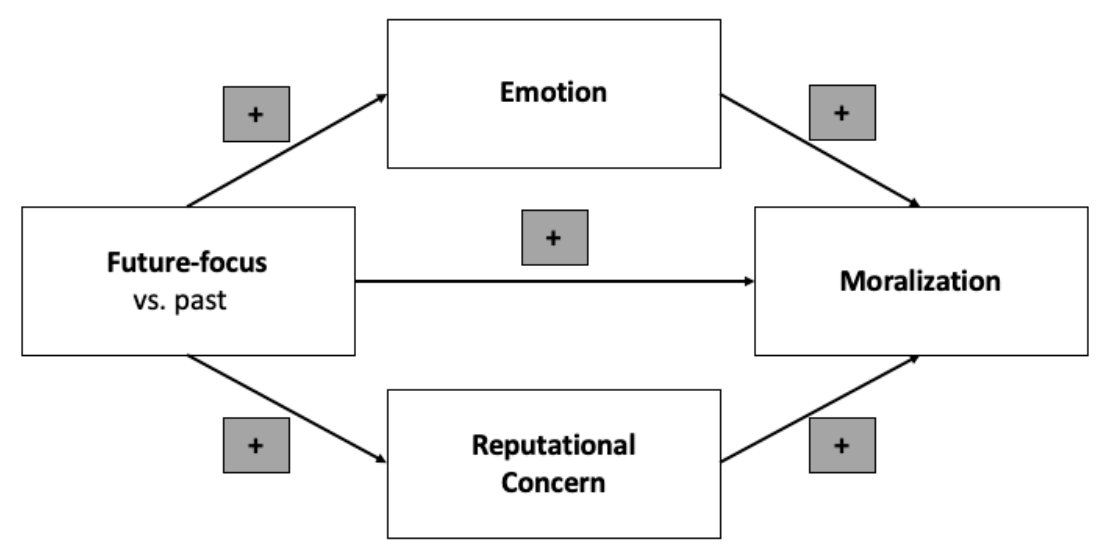

Figure 1. Conceptual model. Thinking about the future (vs. the past) should have a main effect on moralization (i.e., favoring stronger praise and reward for good actions, and stronger blame and punishment for bad actions). Second, people moralize their future actions more than their past actions because the mediating mechanisms of emotion and/or reputational concern are stronger in the future perspective as well. 


\section{Study 1: Harmful and Helpful Choices in the Past vs. Future}

Study 1 provided the first test of the future moralization hypothesis, which is our main prediction across all experiments: People will call for greater rewards and punishments for future than past moral actions. Participants were induced to think about either the future or the past, and then they were asked whether they should experience consequences for morally good and bad actions occurring in the time frame they had been contemplating. If the purpose of moral judgment is to increase one's own positive future outcomes, then they should call for future virtuous actions to be rewarded more than past ones, while they should indicate that future misdeeds deserve less punishment than past ones. In contrast, if morality is about securing a virtuous future all around, participants should indicate that future misdeeds should be punished severely, just as future virtuous actions should be rewarded (and in both cases, more than past actions).

\section{Method}

Participants. Following two university lectures in medicine and organizational science at a Norwegian university, we recruited 194 student participants $\left(N_{\text {future }}=95, N_{\text {past }}=\right.$ 99; 142 female; age $M=22.3$ years). Each participant was compensated with a gift card at a local coffee shop worth approximately \$3. Using the G*power software (Faul, Erdfelder, Lang, \& Buchner, 2007), a sensitivity analysis showed that this sample provided $80 \%$ power to detect a main effect in the magnitude of $d=.40, p<.05$ (two-tailed).

Materials and procedure. Participants agreed to stay in their seats after a lecture to fill out a short questionnaire about "personality and attitudes." They were randomly assigned to a past or a future condition (five years ahead or five years back in time), in a betweensubjects design with two conditions. The first part of the manipulation was administered on 
the first page, instructing participants to stop for a moment and think either about their future, five years ahead from today, or think about their past, five years back.

After participants completed this instruction, the subsequent page of the questionnaire contained two questions that manipulated the direction of time perspective in their wording. The headline stated "In the future [past] - in the next [past] 5 years of your life:". Participants then answered two questions designed to measure moral responsibility: "To what degree do you think you should experience negative consequences (punishment) for the choices you will make [have made] that are [were] harmful or unpleasant for other people?" and "To what degree do you think you should experience positive consequences (reward) for the choices you will make [have made] that are [were] beneficial or helpful for other people?" Both questions were answered on a 11-point response scale ranging from 0 (not at all) to 10 (very much so). As a manipulation check, the last item on the questionnaire asked whether the survey questions had pertained to the past or the future.

\section{Results}

Manipulation check. In the future condition, $88.2 \%$ of participants reported that the questions were indeed about the future, and in the past condition $85.9 \%$ reported that the questions were about the past. The difference in proportions was significant, $\chi^{2}(2, N=139)=$ $104.45, p<.001$. Note that 53 of the 194 participants did not respond to this question, possibly by mistake as it was placed on the last page of the questionnaire. Nonetheless, it seems that participants heeded the manipulation correctly.

Moralization: punishment and reward. An independent $t$-test revealed that participants in the future condition recommended more punishment for harmful choices than participants in the past condition $\left(M_{\text {future }}=6.85, S D=2.24\right.$ vs. $\left.M_{\text {past }}=5.34, S D=2.91\right)$. The difference was statistically significant, and the estimated effect size was medium, $t(192)=$ 
4.05, $p<.001, d=.58$. Participants in the future condition also called for more reward for helpful choices than participants in the past condition $\left(M_{\text {future }}=6.37, S D=1.97\right.$ vs. $M_{\text {past }}=$ $5.16, S D=2.35)$. The difference was significant, and the effect size was medium, $t(192)=$ $3.88, p<.001, d=.55$. We note that both of these results remained robust when excluding the 18 participants who failed the manipulation check. Thus, in line with the future moralization hypothesis, people prescribed more punishment for harmful choices and more reward for helpful choices (see fig. 2).

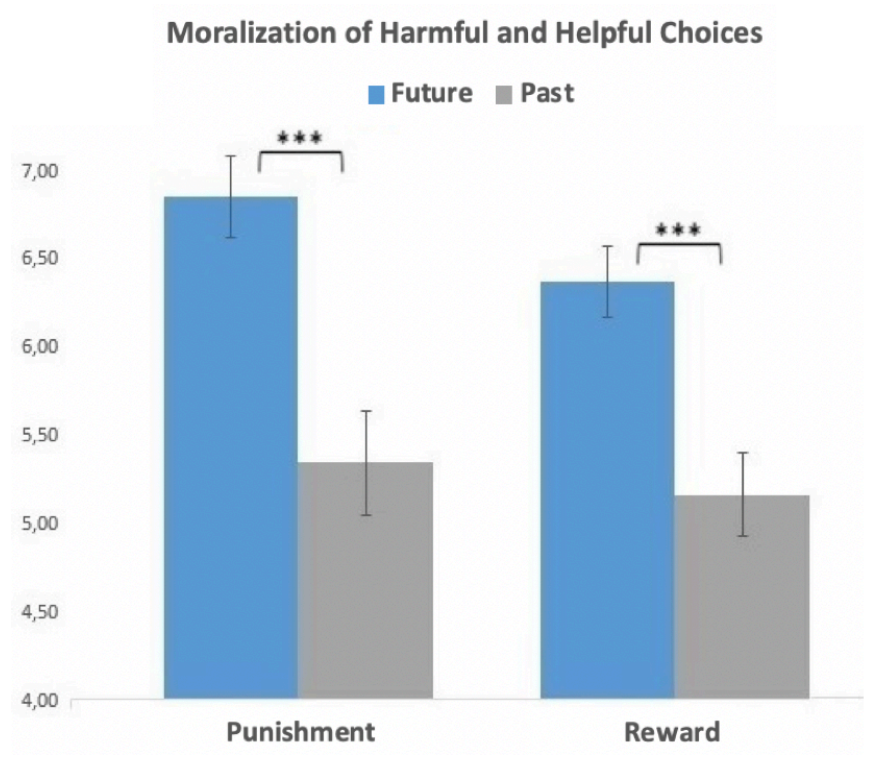

Figure 2. Study 1. Participants prescribed more punishment for harmful choices and more reward for helpful choices to themselves in the future than in the past. Error bars indicate standard error. ${ }^{* * *} p<.001$. Both outcome variables were measured using a 0-10 response scale.

\section{Discussion}

Participants called for more reward for their future virtuous actions than for their past virtuous actions. Perhaps that is not surprising, insofar as the future is valued more than the past (Caruso, Gilbert, \& Wilson, 2008), and people want a pleasant future. More surprising was the finding that participants thought they should receive more severe punishments for their own future misdeeds than for their past ones. These findings extend those of Caruso (2010) to show that the self does not receive special treatment. Moral judgments and 
outcomes are subjectively enhanced in connection with future actions. The seeming implication is that morality is focused on the future, possibly with the goal of improving everyone's behavior — very much including one's own.

Several alternative explanations are possible, however. One is based on optimism and moral progress. Abundant evidence indicates that people expect their lives to be better in the future than in the past (e.g., Weinstein, 1980; for recent review, see Shepperd, Klein, Waters, \& Weinstein, 2013). Although this would not necessarily prompt people to call for intensified punishment for future misdeeds, it might make them less worried about suffering those aversive consequences. We also note that evidence has accumulated that humankind has slowly moved toward broadly more virtuous action and interaction patterns (for review, see Pinker, 2011). If the present is indeed better than the past, it may be appropriate to use higher standards for judging future actions relative to past actions, and to judge oneself accordingly. Study 1 did not measure expectancies about future behavior, but the subsequent studies rectified this to determine whether a pattern of optimism would contribute to judging one's future actions by higher standards than one's past actions.

A related possibility arises given the relatively young age of the sample. The average participant in this study was 22 years old, and so the past condition extended back to age 17 , while the future extended to age 27 . It may be normative to expect morally better behavior from a 27-year-old than from a 17-year-old. Quite plausibly, many people think it is appropriate to tolerate and forgive some teenage misbehavior while also expecting an adult to have outgrown such patterns. Therefore, our further research recruited somewhat older samples and narrowed the time frame to one year rather than five years from the present.

A third possibility for alternative (or supplementary) explanation comes from Bandura's (1999) work on moral disengagement. Bandura suggests that when people perform immoral actions, they disconnect themselves from the moral implications of their work. We 
were not able to study processes involved in actually committing immoral actions, so our work should not be considered an important test of his theory. However, it is possible that participants in Study 1 responded to our questions by disengaging from the wrongness of past behaviors. The past is more constrained by reality than the future, so people would know whether they have or have not performed such actions when thinking back. To address this concern, we included questions about personal relevance in all studies to assess whether this was a factor contributing to future moralization.

\section{Study 2: Bad Deeds in the Past Versus the Future}

We designed Study 2 to extend Study 1 in several ways, in addition to providing a conceptual replication of the future moralization of bad behaviors. We recruited an older sample than the undergraduate population used in Study 1, and the time frame was narrowed from five years to one year in either direction (past or future). Thus, rather than comparing one's moral misdeeds at 17 and 27 years of age, the study compared them at 33 and 35 years of age (on average), on the assumption that most people would expect less moral maturation in that short period of early middle age than during the decade starting in the later teen years. Moving the data collection from a Norwegian university campus to a U.S. online setting also enabled us to assess generalizability. Psychological processes vary in their level of context sensitivity, which in turn may influence reproducibility of some research findings more than others (Van Bavel, Mende-Siedlecki, Brady, \& Reinero, 2016). We acknowledge the importance of contextual factors by testing our general hypothesis in a series of experiments that combine several samples from different settings.

Study 2 also specified the content of the moral actions somewhat more precisely. Some evidence shows that people experience stronger emotional reactions with highly abstract, meaningful thinking than with more concrete thinking (e.g., Vallacher \& Wegner, 
$1985,1987)$, which is reflected in people's shift to concrete thinking when they want to escape negative emotion (e.g., Baumeister, 1990, 1991). Study 1 used only an abstract and vague allusion to making choices that helped or harmed other people, so we wanted to explore whether the effect would translate to a more concrete format as well. Therefore, Study 2 instructed participants to think specifically about "moral shortcuts." These were defined as relatively minor transgressions, but the instructions emphasized that the accumulated impact of many such small transgressions was often greater than that of more severe but much rarer misdeeds. Study 2 also provided several examples of these moral shortcuts, so that all participants would start with similar assumptions about the scale of the misdeeds being discussed. Moral shortcuts were selected on the basis of Mazar, Amir, and Ariely's (2008) finding that extreme cheating and other extreme misbehavior are fairly rare, whereas minor accounts of cheating are relatively common (for cross-societal data, see Gächter \& Schulz, 2016). We wanted to capture acts that people could imagine themselves doing, so we selected these relatively minor and common infractions rather than more heinous crimes.

More important, Study 2 aimed to illuminate the causal process behind the future moralization effect found in Study 1. It asked for anticipated emotional reactions, including guilt, which could mediate the effect. It also tested moral optimism as another possible explanation by measuring whether people thought that they would take fewer moral shortcuts in the future than they had done in the past. Last, it checked for moral disengagement by asking people whether they found the questionnaire material personally relevant.

\section{Method}

Participants. We recruited 160 online participants $\left(N_{\text {future }}=80, N_{\text {past }}=80 ; 94\right.$ female, age $M=34.5$ years) from Amazon Mechanical Turk (MTurk) to participate in a brief study on “attitudes and decisions" in exchange for \$0.40. Using the G*power software (Faul, 
Erdfelder, Lang, \& Buchner, 2007), a sensitivity analysis showed that this sample provided $80 \%$ power to detect a main effect of the magnitude $d=.45, p<.05$ (two-tailed). Thus, Study 2 was powered to detect a minimum effect that was roughly $80 \%$ of the average effect size observed in Study $1(d=.57)$.

Materials and procedure. Participants were randomly assigned to a past or a future condition (one year ahead or one year back in time) in a between-subjects design with two conditions. The manipulation of time perspective consisted of how the questionnaire was introduced on the first screen and the time orientation in the question wording. The following text introduced the questionnaire: "Did you know that 'moral shortcuts' cause much more harm to honest, good people than the few extreme cases of moral corruption such as finance scams and violent crime? Taking a moral shortcut is what we do when we behave in a slightly dishonest, unfair or irresponsible way to benefit ourselves or to make our lives more convenient. It is very common." The instructions went on to list examples, including minor misreporting of taxes, unwarranted absence from work on short notice, and drinking too much alcohol before driving. After participants had confirmed that they understood what was meant by a moral shortcut, they were instructed to think either one year ahead (future condition) or one year back (past condition) when answering the questions that followed.

Below the headline "During the next [past] year," we measured negative emotion with two items, feeling bad and feeling guilty: "Do you think [would you say] that taking moral shortcuts will make [has made] you feel bad?" and "Do you think [would you say] that taking moral shortcuts will make [has made] you feel guilty?" On the next screen, we also measured moral accounting with two items, using the same headline as in the emotion measure, but this time as ratings of blame and prescription of appropriate punishment: "Do you think [would you say] that it will be [would be] blameworthy for you to take moral shortcuts?" and "Do you think [would you say] that it will [would] be fair if you receive [received] punishment for 
taking moral shortcuts?" Participants also reported actual behavior either in the future (predicted) or in the past (remembered): "Do you think [would you say] that you will ever take [have ever taken] some form of moral shortcut at any point in time?"

For all these measures, we used an 11-item response scale ranging from 0 (not at all) to 10 (very much), except for the last question about moral behavior, which was anchored by 0 (never) and 10 (very often). We measured personal relevance by asking participants whether the questions about moral shortcuts applied to them $(1=$ not at all; $2=$ yes, somewhat; $3=$ yes, very much). We included the last two measures to control for moral optimism (expectation of improving in the future) and temporal differences in personal relevance of the questions asked, in case a systematic difference on any of these domains could explain the effect. On the last screen, we asked participants whether the survey questions had pertained to the past or the future, as our manipulation check.

\section{Results}

Manipulation check. Using the same manipulation check as in Study 1, we found that $86.3 \%$ of the participants in the future condition reported that the questions were about the future, and $93.8 \%$ in the past condition reported that the questions were about the past. This difference in proportions was highly significant, $\chi^{2}(2, N=158)=139.45, p<.001$. The vast majority of participants understood the manipulation correctly.

Moralization: blame and punishment. The correlation between ratings of blame and prescriptions of punishment was high $(r=.74, p<.001)$, so we collapsed these variables into a single index of moralization (Cronbach's $\alpha=.85$ ). An independent $t$-test revealed that participants in the future condition assigned more severe blame and punishment to themselves for taking moral shortcuts than participants in the past condition $\left(M_{\text {future }}=6.29, S D=2.46 \mathrm{vs}\right.$. $M_{\text {past }}=5.11, S D=2.62$ ), who responded to identical questions in retrospect. The difference 
MORALIZING THE FUTURE

was statistically significant, and the estimated effect size was medium, $t(158)=2.94, p=$ $.004, d=.46$.

Emotion: feeling bad and feeling guilty. The correlation between ratings of feeling bad and feeling guilty was very high $(r=.90, p<.001)$, so we combined these variables into an index of general negative emotion (Cronbach's $\alpha=.94$ ). Participants in the future condition predicted experiencing a stronger negative emotion over future moral shortcuts than the retrospective reports of participants in the past condition $\left(M_{\text {future }}=6.58, S D=2.55\right.$ vs. $M_{\text {past }}=$ 4.94, $S D=2.94)$. The difference was statistically significant, and the estimated effect size was medium, $t(158)=3.76, p<.001, d=.60$.

Mediation analysis. By conducting a simple mediation analysis in PROCESS (Model 4), we found that anticipated emotion mediated the effect of future-focus on the moralization index (see fig. 3). Thinking about bad deeds in the future produced stronger negative emotion than thinking about similar misdeeds from the past ( $a=1.64, p<.001)$, and stronger negative emotion was associated with an increase in severity of blame and punishment to self $(b=.49$, $p<.001)$. This indirect effect $(a b=.80)$ was statistically significant; a bias-corrected bootstrap confidence interval based on 10,000 bootstrap samples did not include the value 0 $(\mathrm{CI}=.35,1.35)$. The total effect of future-focus on moralization was significant $(c=1.18, p=$ $.004)$, while there was no evidence of a direct effect independent of the emotional mechanism $\left(c^{\prime}=.38, p=.291\right)$. The proportion of the mediated effect (indirect effect/total effect) was .68 , suggesting that the emotional mechanism accounts for approximately two-thirds of the prospective effect on moral accountability for bad deeds. Thus, negative emotion was stronger in prospect than in retrospect, which in turn led people to assign more blame and punishment to themselves for anticipated bad deeds in the future than for actual bad deeds from the past.

Moral optimism and personal relevance. The moral optimism explanation suggests that people think their future behavior will be more virtuous than their past behavior. Contrary 
to this explanation, however, we found no significant difference between participants' rated frequency of past moral shortcuts and anticipated frequency of future moral shortcuts $\left(M_{\text {future }}\right.$ $\left.=5.78 v s . M_{\text {past }}=5.48, p=.480\right)$. Another possible explanation was personal relevance, but the vast majority of the participants in both conditions rated the questions about moral shortcuts as either somewhat relevant or very relevant to them $(88.7 \%$ in the future condition and $85 \%$ in the past condition). The minor difference in proportions between conditions was not significant, $\chi^{2}(2, N=158)=2.39, p=.303$. The results were practically unchanged when we excluded participants who rated the question as irrelevant.

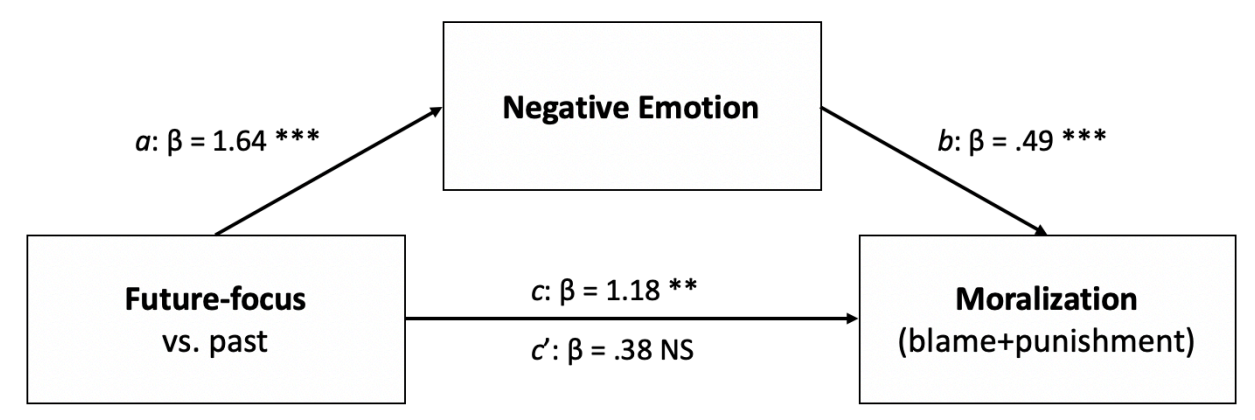

Figure 3. Statistical mediation diagram for Study 2. Thinking about one's bad deeds in the future generated stronger negative emotion than thinking about one's bad deeds from the past. Stronger negative emotion mediated the tendency to moralize future actions more than past actions (i.e., calling for more blame and punishment).

\section{Discussion}

Study 2 replicated the finding that morality is about the future: People think that their own future misdeeds should be punished more harshly than their past ones. More important, Study 2 made progress toward elucidating the process underlying future moralization. In particular, anticipated guilt and other negative emotion appear to be important contributors. People expected to feel more guilt and other bad feelings in connection with future moral shortcuts than they recalled feeling over past ones. These emotions mediated the assignment of greater blame and punishment to self in the future. 
Other explanations were not supported. It was possible that Study 1's findings were affected by comparing teenage and adult misbehavior, but Study 2 compared past and future actions that were much closer in time than those of Study 1. Moreover, for the sample average, both past and future actions occurred during participants' mid-30s. Thus, the effect is not a result of comparing teenagers to adults.

There was also no sign of moral optimism, that is, people did not expect their future selves to be more virtuous than their past selves. Participants' anticipated frequency of moral shortcuts in the next year was about the same as their reported frequency during the past year. Also, there was no sign of differential personal relevance, as the moral disengagement hypothesis might have predicted. Most participants thought that the issues and questions were relevant to their own lives and experiences, and there was no difference by condition.

\section{Study 3: Good Deeds in the Past Versus Future}

Study 1 included both virtuous and immoral actions. Study 2 focused on the immoral ones. In parallel, Study 3 undertook to replicate future moralization of virtuous actions as well, with similar refinements. Specifically, we instructed participants to contemplate the category of "doing good deeds" and provided specific examples of such deeds. We measured anticipated versus recalled emotion, as well as personal relevance. The study also provided another test of the moral optimism explanation by comparing expected frequency of good deeds in the coming year against reported frequency of having done them in the preceding year. As in the other studies, the main hypothesis was that people would moralize their future actions more than similar actions in the past. Specifically, we predicted that people would assign themselves more praise and reward for their good deeds in the future. Furthermore, we predicted that anticipated emotion would once again function as a moral mechanism and mediate this effect. 


\section{Method}

Participants. We recruited 161 participants $\left(N_{\text {future }}=78, N_{\text {past }}=83 ; 87\right.$ female, age $M$ = 34 years) from an MTurk online sample to participate in a brief study on "attitudes and decisions" in exchange for $\$ 0.40$. As in Study 2, a sensitivity analysis in G*power (Faul, Erdfelder, Lang, \& Buchner, 2007) showed that this sample provided $80 \%$ power to detect a main effect of the magnitude $d=.45, p<.05$ (two-tailed).

Materials and procedure. The structural design of Study 3 was identical to that of Study 2. We changed the moral category in question from negative (taking moral shortcuts) to positive (doing good deeds) and adjusted the valence of the measures accordingly, by changing the emotion scales from feeling bad and guilty to feeling good and proud. Likewise, we changed the moralization scales from prescription of blame and punishment to praise and reward.

Participants were randomly assigned to a past or a future condition (one year ahead or one year back in time) in a between-subjects design with two conditions. As in the prior studies, the manipulation of time perspective consisted of how the questionnaire was introduced on the first screen and the time orientation in the headline and question wording. The following text introduced the questionnaire: "Did you know that all the small 'good deeds' in everyday life are helping much more people in need than if people did nothing at all? Doing a good deed is what we do when we pay a small cost to help or benefit others. It is very common." Giving money to charities, helping friends and family, and donating blood were listed as examples of good deeds. After participants had confirmed that they understood what was meant by a good deed, they were instructed to think either one year ahead (future condition) or one year back (past condition) when answering the questions that followed.

We measured positive emotions in connection with past or future good deeds with two items on a scale from 0 (not at all) to 10 (very much): the nonspecific feeling good and the 
more specific feeling proud. "During the next [past] year: Do you think [would you say] that doing good deeds will make [have made] you feel good?" and "Do you think [would you say] that doing good deeds will make [have made] you feel proud?" We also measured moralization with two items on a similar scale (0-10): praise and reward. "Do you think [would you say] that it will be [would be] praiseworthy for you to do good deeds?" and "Do you think [would you say] that it will [would] be fair if you receive [received] reward for doing good deeds?" Except for the change in valence from negative to positive, the question wording of these four items was kept identical to that of Study 2.

Then participants rated their actual frequency of doing good deeds, either as anticipated in the coming year or as recalled from the past year $(0=$ never, $10=$ very often $)$. "During the next [past] year: Do you think [would you say] that you will ever do [have ever done] some form of good deed?" This scale was also the same as in Study 2. As the manipulation check, a single item on the last screen asked participants whether the survey questions had pertained to the past or the future.

\section{Results}

Manipulation check. All participants in the past condition reported that the questions were about the past, and all but one participant in the future condition reported that the questions they had answered were about the future (98.7\%).

Moralization: praise and reward. The correlation between rated praise and appropriate reward was high $(r=.61, p<.001)$, so we combined the two items into an index of moralization (Cronbach's $\alpha=.76)$. An independent $t$-test revealed that participants in the future condition assigned themselves more praise and reward for doing good deeds than participants in the past condition $\left(M_{\text {future }}=5.76, S D=2.63\right.$ vs. $\left.M_{\text {past }}=4.93, S D=2.65\right)$. The 
MORALIZING THE FUTURE

difference was significant, and the estimated effect size was moderate, $t(159)=1.99, p=.048$, $d=.31$.

Emotion: feeling good and feeling proud. Feeling good and feeling proud were highly correlated $(r=.87, p<.001)$, so we combined them to form an index of general positive emotion (Cronbach's $\alpha=.93$ ). Participants in the future condition indicated stronger positive emotion than participants in the past condition $\left(M_{\text {future }}=8.44, S D=1.79\right.$ vs. $M_{\text {past }}=$ $7.77, S D=2.34)$. The difference was statistically significant, and the estimated effect size was moderate, $t(159)=2.07, p=.040, d=.32$.

Mediation analysis. As in Study 2, using simple mediation analysis in PROCESS (Model 4), we found that anticipated emotion mediated the effect of future-focus on the moralization index (see fig. 4). Thinking about good deeds in the future produced stronger positive emotion than thinking about similar good deeds from the past $(a=.68, p=.042)$, and stronger positive emotion was associated with an increase in assignment of praise and reward to self $(b=.37, p<.001)$. This indirect effect $(a b=.25)$ was statistically significant; a biascorrected bootstrap confidence interval based on 10,000 bootstrap samples did not include the value $0(\mathrm{CI}=.02, .56)$. The total effect of future-focus on appropriate praise and reward was significant $(c=.83, p=.048)$, while there was no evidence for a direct effect independent of the emotional mechanism $\left(c^{\prime}=.58, p=.155\right)$. The proportion of the mediated effect (indirect effect/total effect) was .30, suggesting that the emotional mechanism accounts for approximately one-third of the prospective effect on moralization of good deeds. Thus, positive emotion was stronger in prospect than in retrospect, which in turn led people to assign more praise and reward to themselves for anticipated good deeds in the future than for actual good deeds from the past.

Moral optimism and personal relevance. Participants in the future condition predicted that they would do more good deeds in the coming year than those in the past 
condition rated they had done in the previous year $\left(M_{\text {future }}=8.44, S D=1.89\right.$ vs. $M_{\text {past }}=7.77$, $S D=2.12), t(159)=2.09, p=.038, d=.33$. Therefore, we explored the causal process in a simultaneous mediation model in PROCESS (Model 4) to test whether optimism could uniquely explain the future moralization effect when we included anticipated emotion in the model as well. The mediation effect of positive emotion remained significant, as indicated by a $95 \%$ CI that did not include the value $0(.01, .63)$, while no mediation effect occurred for moral optimism $(\mathrm{CI}=-.20, .20)$. Thus, moral optimism was present, but it did not mediate the prospective effect on praise and reward.

Furthermore, as in Study 2, the vast majority of participants in both conditions rated the questions about doing good deeds as either somewhat relevant or very relevant to them ( $97.4 \%$ in the future condition and $97.6 \%$ in the past condition). The minor difference in proportions between conditions was not significant, $\chi^{2}(2, N=159)=1.75, p=.417$. Thus, there was no evidence to suggest that temporal differences in personal relevance was a viable alternative explanation either.

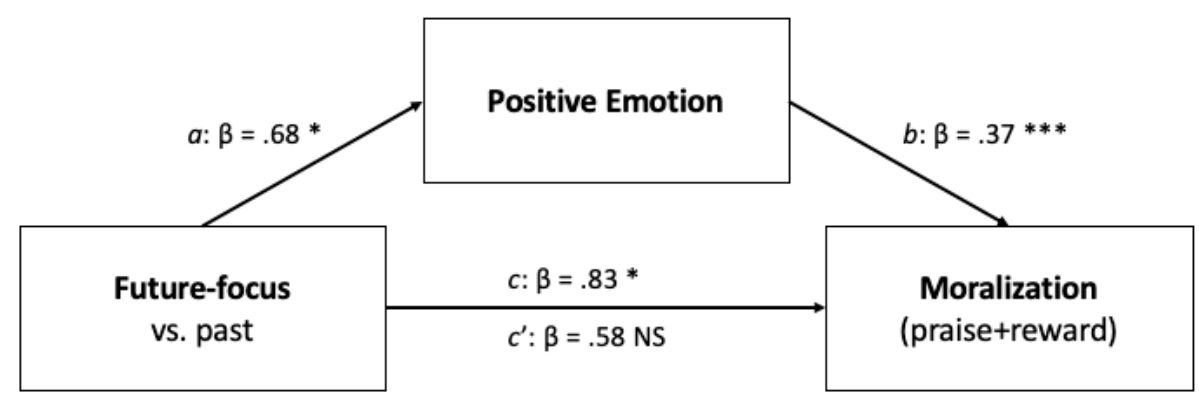

Figure 4: Statistical mediation program for Study 3. Thinking about one's good deeds in the future generated stronger positive emotion than thinking about one's good deeds from the past. Stronger positive emotion mediated the tendency to moralize future actions more than past actions (i.e., calling for more praise and reward).

\section{Discussion}

The findings regarding virtuous behavior in Study 3 were largely parallel to those regarding immoral actions in Study 2, so the future moralization effect was again successfully 
replicated. Participants rated higher levels of praise and reward to themselves as appropriate for future than past good deeds. They expected future good deeds to evoke more positive emotions than past good deeds, and these emotions mediated the tendency to moralize the future more than the past. Personal relevance did not differ between past and future.

The primary difference between this study and the other studies was in the results for moral optimism. Participants expected to do more good deeds in the future year than others had done in the past year, whereas Study 2 found no evidence of this moral optimism. Even so, this was less relevant to the main findings, insofar as moral optimism failed to mediate the main effect of greater praise and reward for future good deeds than for past good deeds.

A closer inspection of the findings also revealed that Study 2's effects were slightly stronger than Study 3's (smaller $p$-value and larger effect size). This finding might reflect chance variation, but it could also signify that the future moralization pattern is stronger for morally bad than morally good acts.

\section{Study 4: A Direct and Extended Replication of Studies 2 and 3}

To assess the robustness and reproducibility of our findings, we designed Study 4 as a direct replication of Studies 2 and 3. That is, for both good and bad actions alike, we wanted to replicate the main effect of future moralization, and to demonstrate again that the effect is driven by anticipated emotion through mediation. Procedures were kept the same, with two small exceptions. First, we added reputational concern as another possible mediating variable, to broaden the scope from internal (emotion) to social mechanisms. Reputational concern plays a central role in cooperation and morality (for review, see Rand \& Novak, 2013), and is in itself a delayed consequence of one's actions. Reputational concerns may therefore account for the greater moral concern about future than past actions. Presumably, past actions have already had whatever effect they are going to have on one's reputation, but future actions 
could still affect a person's reputation for better or worse. We added this measure after all the other measures, so it would not affect the other measures or change the sequence.

Second, all participants completed a brief mood scale at the beginning of the experiment, so we could control for initial differences in mood. We did this before participants received the manipulation of time perspective, so again it would not alter the flow or sequence. We added this assessment from the speculation that initial mood might affect moral judgment.

\section{Method}

Participants. Assuming a minimum effect size of $d=0.40(\mathrm{p}<.05$, two-tailed $)$, we aimed for a sample size of 100 participants per condition to detect the effect with $80 \%$ power (Cohen, 1988; Faul, Erdfelder, Lang, \& Buchner, 2007). We therefore recruited a total sample of 400 participants $\left(N_{\text {future }}=196, N_{\text {past }}=203 ; 209\right.$ female, age $M=37$ years $)$ from MTurk for a brief online study on "attitudes and decisions" in exchange for $\$ 0.40 .200$ participants were assigned to Study $4 \mathrm{a}$ on bad deeds $\left(N_{\text {future }}=98, N_{\text {past }}=102\right)$, and the remaining 200 participants were assigned to Study $4 \mathrm{~b}$ on good deeds $\left(N_{\text {future }}=98, N_{\text {past }}=102\right)$.

Materials and procedure. Participants were randomly assigned to Study 4a (replication of Study 2: moral shortcuts in the future or past) or Study $4 \mathrm{~b}$ (replication of Study 3: replication of good deeds in the future or past), in a between-subjects design with four conditions in total. Before receiving the manipulation of time perspective, all participants completed the Positive and Negative Affect Scale (Watson, Clark, \& Tellegen, 1988). As in Studies 2 and 3, the manipulation of time perspective consisted of how the questionnaire was introduced and the time orientation in question wording. After reading and confirming the stimuli material about moral shortcuts or good deeds, all participants reported prospective or retrospective emotion (Study 4a: feeling bad and feeling guilty; Study $4 \mathrm{~b}$ : feeling good and 
feeling proud) and rated appropriate levels of moral consequences (Study 4a: blame and punishment; Study 4b: praise and reward). We used the same measures as in Studies 2 and 3.

After completing the replication procedure, all participants responded to a new twoitem measure of reputational concern. This appeared on the first screen following the moralization measures (blame and punishment, or praise and reward). Study 4a used the following items: In the future condition, "During the next year: Do you think that taking moral shortcuts will be bad for your reputation among friends and family?" and "Do you think that taking moral shortcuts will put you at risk of being excluded from the groups and communities you belong to?"; in the past condition, "During the past year: Would you say that taking moral shortcuts has been bad for your reputation among friends and family?" and "Would you say that taking moral shortcuts has put you at risk of being excluded from the groups and communities you belong to?"

In Study 4b, the wording was the same except that "taking moral shortcuts" was replaced with "doing good deeds," and of course the possible effects were described as good rather than bad. Future condition: "During the next year: Do you think that doing good deeds will be good for your reputation among friends and family?" and "Do you think that doing good deeds will increase your chances of being fully accepted in the groups and communities you belong to?" Past condition: "Would you say that doing good deeds has been good for your reputation among friends and family?" and "Would you say that doing good deeds has increased your chances of being fully accepted in the groups and communities you belong to?" We used the same 11-point scale (ranging from 0-10) as in the prior studies. On the last screen we included the same manipulation check as in the previous studies, asking participants whether the survey questions were about the future or the past. 
MORALIZING THE FUTURE

\section{Results: Study 4a (Bad Deeds)}

Manipulation check. Ninety-eight percent of participants in the future condition reported that the questions were about the future, and $95.1 \%$ of participants in the past condition reported that the questions were about the past. The difference in proportions was significant, $\chi^{2}(2, N=200)=173.14, p<.001$.

Moralization: blame and punishment. The correlation between ratings of blame and appropriate punishment was high $(r=.69, p<.001)$, so again we combined them for an index of moralization (Cronbach's $\alpha=.82$ ). An independent $t$-test revealed that participants in the future condition called for greater blame and punishment for taking moral shortcuts than participants in the past condition $\left(M_{\text {future }}=6.88, S D=2.49\right.$ vs. $\left.M_{\text {past }}=5.70, S D=2.75\right)$. The difference was statistically significant, and the estimated effect size was medium, $t(198)=$ $3.19, p=.002, d=.45$.

Emotion: feeling bad and feeling guilty. Again, feeling bad and feeling guilty were highly correlated $(r=.90, p<.001)$, so we combined them (Cronbach's $\alpha=.94)$. Participants in the future condition indicated stronger negative emotion in their prospective reports than the retrospective reports of participants in the past condition $\left(M_{\text {future }}=7.05, S D=2.88 \mathrm{vs}\right.$. $\left.M_{\text {past }}=5.61, S D=3.30\right)$. The difference was statistically significant, and the estimated effect size was medium, $t(198)=3.28, p=.001, d=.46$.

Reputational concern. The correlation between explicit reputational consequences and perceived risk for social exclusion was high $(r=.84, p<.001)$, so we collapsed these two measures into a general index for reputational concern (Cronbach's $\alpha=.91$ ). Participants in the future condition indicated more reputational concern in their prospective judgments than the retrospective judgments of participants in the past condition $\left(M_{\text {future }}=5.76, S D=3.14\right.$ vs. $\left.M_{\text {past }}=3.91, S D=3.06\right)$. The difference was statistically significant, and the estimated effect 
MORALIZING THE FUTURE

size was medium, $t(198)=4.22, p<.001, d=.60$. This is novel evidence of the importance of reputational concern in moral prospection.

Mediation analysis. Using simultaneous mediation analysis in PROCESS (Model 4), we found that anticipated emotion and reputational concern each uniquely mediated the effect of future-focus on the index of moralization (see fig. 5). In the first path, thinking about bad deeds in the future produced stronger negative emotion than thinking about similar bad deeds from the past $\left(a_{1}=1.85, p<.001\right)$, and stronger negative emotion was associated with calling for more blame and punishment for future misdeeds $\left(b_{1}=.22, p<.001\right)$. This indirect effect $\left(a_{1} b_{1}=.41\right)$ was statistically significant; a bias-corrected bootstrap confidence interval based on 10,000 bootstrap samples did not include the value $0(\mathrm{CI}=.18, .71)$. In the second path, thinking about bad deeds in the future produced stronger reputational concern $\left(a_{2}=1.44, p=\right.$ .001 ), which was likewise associated with calling for higher blame and punishment for future than past misdeeds $\left(b_{2}=.48, p<.001\right)$. This second indirect effect $\left(a_{2} b_{2}=.69\right)$ was statistically significant as well, in that the bootstrapped confidence interval did not include the value $0(\mathrm{CI}=.27,1.15)$.

The total effect of future-focus on moralization was significant $(c=1.18, p=.002)$, while there was no evidence for a direct effect independent of the emotional and reputational mechanisms $\left(c^{\prime}=.08, p=.747\right)$. The proportion of the mediated effect (sum of indirect effects/total effect) was .93 , suggesting that the emotional and reputational mechanisms can account for approximately $90 \%$ of the prospective effect on calling for increased blame and punishment for one's bad deeds. Thus, negative emotion and reputational concern were stronger in prospect than in retrospect, which in turn led people to moralize their future bad deeds to a greater extent than their bad deeds in the past.

Baseline mood. Follow-up analyses using baseline mood (PANAS) yielded little of interest. Positive mood was positively correlated with calling for more blame and punishment 
for future than past misdeeds $(p<.001)$. With mood as a covariate, the primary finding that people moralized their future actions more than past actions remained highly significant $(p<$ .001 ), unlike with good deeds (see Study $4 \mathrm{~b}$ below).

Moral optimism and personal relevance. We found no evidence for moral optimism in Study 4a. Participants in the future condition predicted an almost identical frequency of taking moral shortcuts in the future $\left(M_{\text {future }}=4.98, S D=2.79\right)$ as participants in the past condition reported in retrospect $\left(M_{\text {past }}=4.94, S D=3.31, p=.93\right)$. Assuming that participants in the past condition did not systematically underreport their actual amount of moral shortcuts taken, we excluded moral optimism as an alternative explanation for the effect. As in Studies 2 and 3, there was also no evidence for the moral disengagement explanation. The majority of participants in both conditions rated the questions about moral shortcuts as either somewhat relevant or very relevant to them $(86.7 \%$ in the future condition and $81.4 \%$ in the past condition), and the minor difference in proportions between conditions was not significant, $\chi^{2}(2, N=200)=1.55, p=.462$.

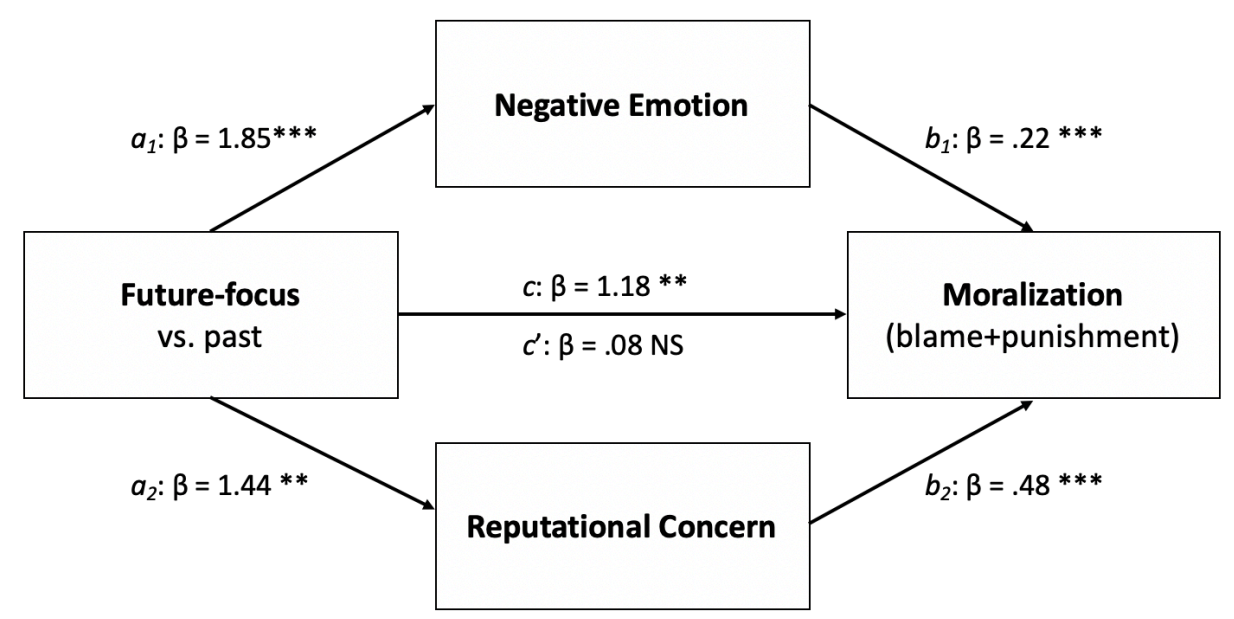

Figure 5. Statistical mediation diagram for Study 4a. Thinking about one's bad deeds in the future generated stronger negative emotion and reputational concern than thinking about one's bad deeds from the past. Stronger negative emotion and reputational concern mediated the tendency to moralize future actions more than past actions (i.e., calling for more blame and punishment). 


\section{Results: Study 4b (Good Deeds)}

Manipulation check. Ninety-eight percent of participants in the future condition reported that the questions were about the future, and $96 \%$ of participants in the past condition reported that the questions were about the past. The difference in proportions was significant, $\chi^{2}(2, N=199)=175.80, p<.001$.

Moralization: praise and reward. The combined ratings of praise and appropriate reward constituted the index of moralization (Cronbach's $\alpha=.77$ ). An independent $t$-test revealed that participants in the future condition thought it appropriate to receive more praise and reward for doing good deeds than participants in the past condition $\left(M_{\text {future }}=6.28, S D=\right.$ 2.54 vs. $\left.M_{\text {past }}=5.46, S D=2.68\right)$. The difference was statistically significant, and the estimated effect size was moderate, $t(197)=2.22, p=.028, d=.31$.

Emotion: feeling good and feeling proud. The items about feeling good and feeling proud constituted an index of general positive emotion (Cronbach's $\alpha=.94)$. Participants in the future condition indicated stronger positive emotion in their prospective reports than the retrospective reports of participants in the past condition $\left(M_{\text {future }}=8.40, S D=2.15\right.$ vs. $M_{\text {past }}=$ $7.46, S D=2.75)$. The difference was significant, and the estimated effect size was moderate, $t(197)=2.69, p=.008, d=.37$.

Reputational concern. The two items measuring explicit reputational consequences and perceived risk for social exclusion were highly correlated $(r=.70, p<.001)$, so we combined them to create an index for reputational concern (Cronbach's $\alpha=.82$ ). Participants in the future condition indicated more reputational concern than participants in the past condition $\left(M_{\text {future }}=6.65, S D=2.50\right.$ vs. $\left.M_{\text {past }}=5.86, S D=2.76\right)$. The difference was statistically significant, and the estimated effect size was moderate, $t(197)=2.11, p=.036, d$ $=.30$. 
MORALIZING THE FUTURE

Mediation analysis. Using simultaneous mediation analysis in PROCESS (Model 4), we found that the effect of future-focus on the moralization of good deeds was uniquely mediated by reputational concern $(\mathrm{CI}=.05, .93)$ but not by anticipated emotion $(\mathrm{CI}=-.10$, .26). However, when we excluded reputational concern from the mediation analysis, the indirect effect of emotion was significant $(.06, .68)$. This is also the way we analyzed the data in Study 3. Thus, we replicated the findings of Study 3 when running an identical analysis, but when reputational concern was included in the model as an unrelated mechanism, anticipated emotion did no longer significantly predict moralization.

After reflection, we speculated that the psychological process might be serial and complementary, especially insofar as emotional impact and reputational concern could easily work together in the process of moralizing future actions. In line with this reasoning, Hayes (2013) recommends serial mediation models when two related mediators are supplementary rather than competing mechanisms. Using serial mediation analysis in PROCESS (Model 6) and placing anticipated emotion as mediator 1 and reputational concern as mediator 2, we found that the effect of future-focus on the moralizing index was indeed mediated through this specific sequence (see fig. 6).

In this serial mediation model, we found that thinking about good deeds in the future activated stronger positive emotion than thinking about similar good deeds from the past $\left(a_{1}=\right.$ $.94, p=.008)$, and stronger positive emotion activated stronger reputational concern $\left(d_{21}=\right.$ $.50, p<.001)$, which in turn led to setting higher levels of praise and reward for one's future good actions $\left(b_{2}=.59, p<.001\right)$. Bootstrapped confidence intervals showed that this specific indirect effect $\left(a_{1} d_{21} b_{2}=.28\right)$ was significant, as indicated by a $95 \% \mathrm{CI}$ that did not include the value $0(.07, .53)$. Moreover, the direct path estimate from emotion to future moralization was very weak and not statistically significant $\left(b_{1}=.05, p=.48\right)$. The total effect of futurefocus on praise and reward ratings was significant $(c=.82, p=.028)$, while there was no 
evidence for a direct effect independent of the emotional and reputational mechanisms $\left(c^{\prime}=\right.$ $.31, p=.305$ ). The proportion of the mediated effect (indirect effect/total effect) was .34 , suggesting that the serial mediation pathway can account for approximately one-third of the prospective effect on judgments of appropriate levels of reward and praise for good deeds.

For exploratory purposes, we also ran an alternative serial mediation model in which we reversed the order of the mediators. In this model, thinking of future good deeds first activates reputational concern, which then activates a stronger emotional response, which in turn directly increases judgments of appropriate praise and reward. We found no support for this model $\left(a_{2} d_{21} b_{1}=.017, \mathrm{CI}=-.05, .09\right)$. Thus, both positive emotion and reputational concern were stronger in prospect than in retrospect, and in that particular sequence (i.e., by serial mediation) they produced the prospective increase in moralization of good deeds (i.e., higher ratings of appropriate praise and reward).

Baseline mood. With mood as a covariate, the primary finding that judgments of appropriate praise and reward were higher for the future than for the past became statistically nonsignificant $(p=.057)$, possibly indicating the relative weakness or context-sensitivity of that effect (in contrast to the blame and punishment judgments of bad deeds, cf. Study 4a).

Moral optimism and personal relevance. We replicated evidence for moral optimism regarding good deeds, but again this effect failed to mediate the moralization judgments when treated as a unique mechanism in a simultaneous mediation model (Model 4). Participants predicted more good deeds in the coming year than other participants reported for the preceding year $\left(M_{\text {future }}=8.55, S D=2.02\right.$ vs. $\left.M_{\text {past }}=7.36, S D=2.62\right), t(197)=3.60, p$ $<.001, d=.51$. However, including this effect in a simultaneous mediation model failed to yield a confidence interval that excluded the value $0(\mathrm{CI}=-.16, .32)$. As in Studies $2-4 a$, there was no evidence for the moral disengagement explanation. The majority of participants in 
both conditions rated the questions about good deeds as either somewhat relevant or very relevant to them ( $98 \%$ in both the future condition and the past condition).

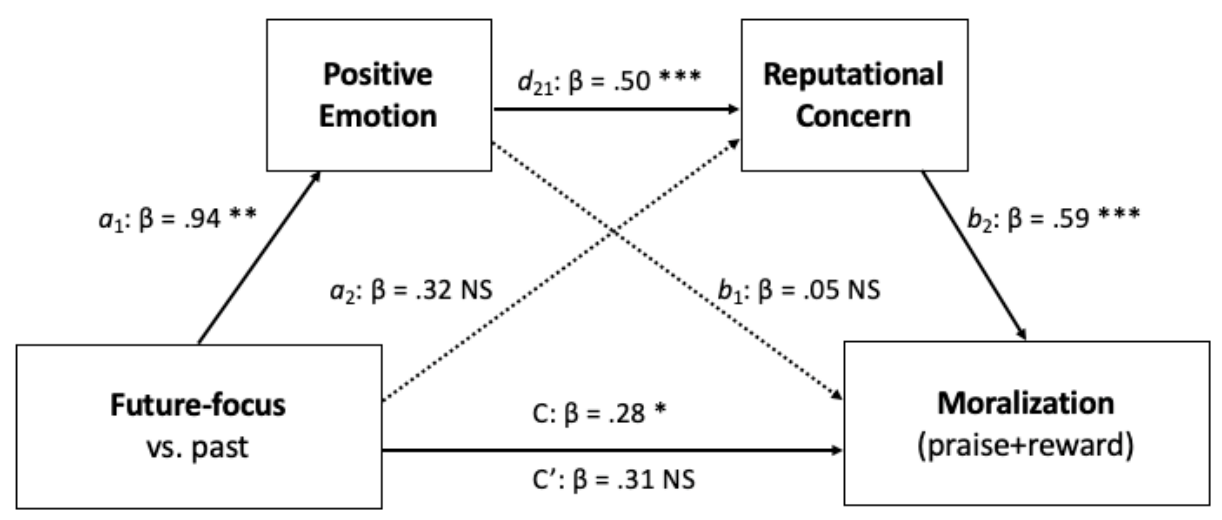

Figure 6. Statistical mediation diagram for Study 4b. Thinking about one's good deeds in the future generated stronger positive emotion and (positive) reputational concern than thinking about one's good deeds from the past. Working together in a serial process, stronger positive emotion and reputational concern mediated the tendency to moralize future actions more than past actions (i.e., calling for more praise and reward).

\section{Discussion}

Study 4 replicated and extended the findings of Studies 1-3. People moralized their future choices to a greater extent than their past choices (for overview, see Figure 7). This was true of both good deeds and (im)moral shortcuts. The effect for good deeds was again weaker than the effect for moral shortcuts. Moral optimism was again irrelevant to immoral action, whereas there were consistent signs of moral optimism in connection with good deeds. Even so, moral optimism failed to mediate the effect of time frame on the moral judgment of appropriate reward and punishment. In contrast, anticipated emotion was again stronger than retrospectively recalled emotion, and the emotional differences did significantly mediate the tendency to moralize future actions more past actions.

Beyond internal replication, the novel contribution of Study 4 was the inclusion of the measure of reputational concern. This also showed a significant difference: Participants thought that the risk of reputation damage and social exclusion was greater in connection with future misdeeds than it had been in the past, and likewise good deeds would enhance their 
reputations and social acceptance more in the future than they had in the past. Reputational concern also mediated the prospective effect on moralization. Indeed, reputational concern was a more powerful mediator than emotion, such that when the two were treated as competing explanations, reputational concern was the only significant mediator. However, additional analyses suggested that this is because emotion and reputational concern were related and actually mediate together. We note that this specific serial mediation pattern was not predicted a priori and therefore should be interpreted with caution.
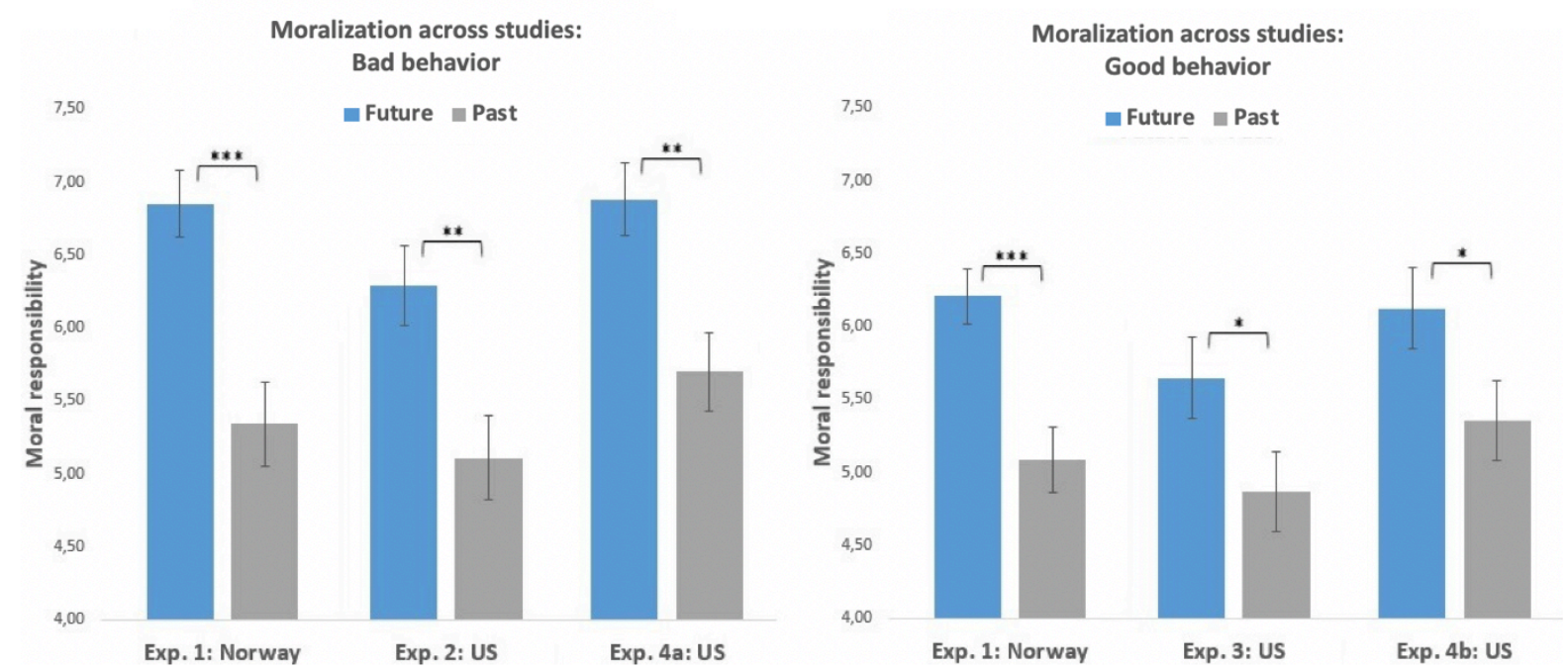

Figure 7. Overview of mean ratings of moralization across all studies (i.e., appropriate blame and punishment for bad actions; appropriate praise and reward for good actions). Error bars indicate standard error. ${ }^{* * *}=p<.001, * *=p<.01, *=p<.05$. All outcome variables were measured using a $0-10$ response scale. Participants called for more extreme moral consequences for both their good and bad behavior in the future, relative to how similar actions were judged in the past (between-subjects).

\section{Study 5: Internal meta-analysis}

Future moralization, or the tendency to call for more extreme moral consequences for one's actions in the future than in the past, is the central finding in this investigation. Specifically, this includes higher levels of blame and punishment ratings for bad actions, and higher levels of praise and reward for good actions. In response to a constructive recommendation from one 
of the reviewers, we conducted an internal meta-analysis of this effect across all four studies and the total sample of 915 participants.

The goal with this analysis was two-fold. First, we wanted to estimate a single metaanalytic effect size across studies. For any paper consisting of multiple experiments with different sample sizes, $\mathrm{p}$-values and effect sizes, a statistical summary of the findings is helpful in evaluating the general strength of the effect. Second, we wanted to examine whether the future moralization effect was moderated by behavior valence (positive vs. negative actions).

\section{Method}

Across all four studies $(N=915)$ and a total of six effect sizes, we conducted an internal meta-analysis of the future moralization effect. The analysis was conducted in JASP (2018) using fixed effects. The basis for the meta-analysis was the standardized effect size (Cohen's d) and the standard error for each effect.

\section{Results}

Main effect: Future moralization. The meta-analytic results showed that the average effect of time perspective on moralization judgments was $d=.45$. This is indicative of a moderate-to-medium effect size, which was highly significant $(\mathrm{Z}=7.36, p<.001$, two-tailed). Thus, in line with the central hypothesis in this investigation, people called for more extreme moral consequences for their future actions than they did for similar actions in the past. See figure 8 for a visual illustration of the results. 


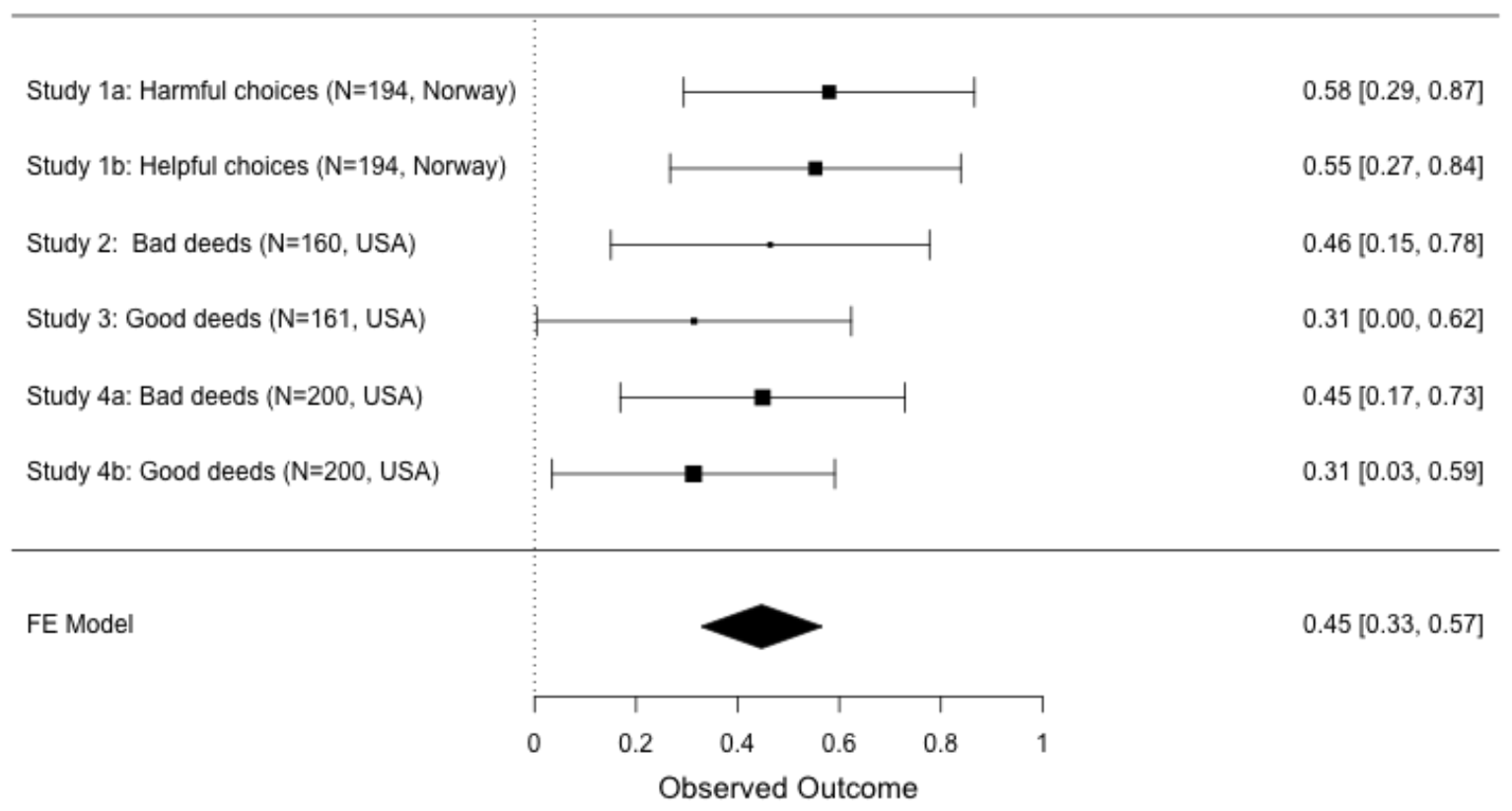

Figure 8. Meta-analytic forest plot of the future moralization effect: Participants called for more extreme moral consequences for their future than past actions, both for good (praise + reward/2) and bad (blame + punishment/2) behaviors. Error bars indicate $95 \%$ confidence intervals, and is stated numerically in the parentheses following each effect size. The meta-analytic effect across all studies is located in the bottom right corner $(\mathrm{d}=0.45)$.

Positive vs. negative events. When analyzed separately, the meta-analytic effect size was larger for negative $(d=.50, \mathrm{CI}=.33, .67)$ than positive $(d=.40, \mathrm{CI}=.23, .56)$ events. Moreover, the meta-analytic forest plot (figure 8) shows that the confidence interval for Study 3 on positive events ("good deeds") includes zero, which means that this particular study does not provide clear evidence for the effect. However, when behavior valence was included as a covariate in the meta-analysis of all six effect sizes across all four studies, valence did not significantly predict moralization judgments $(Z=.85, p=.40)$. Similarly, the test of residual heterogeneity did not identify a significant inconsistency in study outcomes $(\mathrm{Q}=2.25, p=$ $.69)$.

Discussion. In line with a specific recommendation by one of the reviewers, Study 5 was an internal meta-analysis of the future moralization effect in the present investigation (Studies 1-4). The results showed that the meta-analytic effect was moderately strong and highly significant. We also tested the possibility that the effect would be statistically different 
depending on behavior valence, as a purely qualitative assessment of the individual studies might suggest — but we did not find support for this hypothesis. We note that the number of studies is very small for making this type of comparison, suggesting that the statistical power to identify a true moderator is rather low.

\section{General Discussion}

Across four experiments that, in aggregate, used more than 900 participants and were run on two different continents, we found consistent evidence for the future moralization effect: People thought their future choices deserved greater moral consequences than similar choices in the past. That is, the participants called for more blame and punishment for future misdeeds than for past ones, and more praise and reward for future good deeds than for past ones.

Caruso (2010) showed that people judge imagined actions by strangers (including corporations and machines) more stringently when contemplating future than past misdeeds. One way of interpreting those findings is through pure self-interest: People seek a better future for themselves, and getting other people to behave properly is highly conducive to that. (Another explanation is that people believe that others' future misdeeds could adversely affect them, whereas if these actions have already occurred in the past, they know they have not been adversely affected.) However, the self-interest hypothesis would predict that people would tend to excuse their own future misdeeds. After all, being punished is not, by definition, consistent with self-interest. Yet the results provide strong evidence against that view, because people called for more severe condemnation of their own future misdeeds than of their past ones.

The implication is that morality is about the collective future. Our findings (together with those of Caruso [2010]) suggest that the purpose of morality is to move toward a culture 
in which people cooperate effectively on joint projects in a context of mutual trust and fairness. People seem to hold higher standards for future than for past behavior — again, for themselves as well as for others.

The future moralization effect was not due to moral optimism. We found no sign that people expected themselves to desist from future misdeeds any more than they had in the past. We did find limited evidence that they expected to perform more good deeds in the future than in the past, but this did not explain (mediate) why people assigned greater moral consequences to themselves for future than past deeds. The future moralization effect is apparently about what people think should happen rather than what they think will happen. In addition, future moralization cannot be explained based on expecting a more virtuous future and on that basis invoking higher standards. Rather, people judge the future with greater extremity than the past.

In contrast to moral optimism, anticipated emotion and reputational concern did mediate the future moralization effect (i.e., calling for greater moral consequences for future than past actions). As such, these deserve further comment.

Concern for one's reputation is inherently oriented toward the future. Tomasello (2014) has proposed that a crucial and distinctive feature of human evolution was the development of cooperation among non-kin (see also Suddendorf, 2013). As he explains, a species that relies on cooperation to improve survival and reproduction puts an added burden on individuals to make themselves attractive to future potential cooperators. Building a moral reputation is essential to this evolutionary imperative. A recent study measuring behavioral responses to a moral dilemma provided evidence of the early and unique emergence of concern about moral reputation in human children. Five-year-old human children behaved more virtuously when others were watching them than when they were alone, whereas adult chimpanzees acted the same regardless of whether another chimp was watching or not 
(Engelmann, Herrmann, \& Tomasello, 2012). Other studies have combined evolutionary models and economic games to study cooperation and moral behavior (for review, see Rand \& Novak 2013) and have reached similar conclusions about the crucial function of reputational concern (Jordan, Hoffman, Bloom, \& Rand, 2016). For instance, a recent study found that instructing people to focus on the future (vs. the present) made them more generous, because focusing on the future also made them more concerned with maintaining a positive reputation (Sjåstad, 2019). For present purposes, the implication is that concern about one's moral reputation is based on prospective concerns (i.e., the anticipated need to attract cooperative partners). The present findings fit this analysis: The more participants were concerned about their future moral reputation, the more they moralized their future actions in terms of setting appropriate levels of reward and punishment.

The prospective aspect of emotion has received increasing attention from researchers. The present findings fit the affective forecasting pattern (Wilson \& Gilbert, 2005), by which people overestimate future emotions relative to actual experience. Our findings indicated that people overestimated future guilt and pride over moral outcomes, relative to the past. That would also explain why they thought more extreme consequences were appropriate.

One thrust of affective forecasting research is that overestimating future emotional reactions can motivate people to work harder to succeed (Morewedge \& Buechel, 2013), an idea that can be traced back at least to defensive pessimism (Norem \& Cantor, 1986). The present findings suggest that the same logic may apply to moral choice: Overestimating one's likely future guilt, for example, may deter one from misbehaving.

More broadly, a recent meta-analysis of mediation analyses in social psychology found that currently felt emotion was generally not a mediator of judgment or actual behavior (significant in only a small minority of cases) — whereas anticipated emotion, though much less frequently studied, was typically a significant mediator (DeWall et al., 2016). This notion 
fits the theory of emotion as a feedback mechanism, in which people learn to anticipate what actions will bring what emotional outcomes and adjust their behavioral choices accordingly (Baumeister et al., 2007).

Pulling these theoretical strands together, the present study findings suggest that people call for greater moral consequences for future than past moral actions because they anticipate future emotions to be stronger than they recall past ones to have been and because reputational concerns are oriented toward the future. Moreover, anticipated emotion and reputational concern may work together, as our follow-up analyses to Study $4 b$ (on good deeds) suggested. The emotional reactions apparently come first, consistent with the view that moral affect is often quick and automatic, and then drives other cognitive and motivational processes (Haidt, 2001, 2007). In the present case, perhaps, the emotional response is the first signal about the morality of the action, which in turn prompts the person to reflect on the implications for his or her reputation. The greater concern with one's reputation then causes the person to call for greater moral accountability for future than past actions.

Some differences in effect sizes and patterns of effects may be meaningful. In general, we found that the future moralization effect was more robust for bad than good actions. Indeed, controlling for baseline affect knocked the future moralization effect just out of the significant range for good deeds, whereas the effect remained unchanged for immoral actions (Study 4). In contrast, we consistently found moral optimism for good actions but not for bad actions. Bad actions, experiences, stimuli, prospects, and outcomes generally have stronger impact than good ones (Baumeister, Bratslavsky, Finkenauer, \& Vohs, 2001; Rozin \& Royzman, 2001), and this is certainly true for morality. In general, moral rules focus more on prohibiting misdeeds than exhorting virtuous ones. The Ten Commandments in the JudeoChristian tradition, for example, are overwhelmingly about what "thou shalt not" do, with at most two of them somewhat focused on what one should do (Baumeister \& Juola Exline, 
MORALIZING THE FUTURE

1999). Still, our internal meta-analysis did not indicate that the overall strength of effects for bad actions was significantly stronger than for good ones.

\section{Limitations and Future Directions}

The present studies measured moral judgments about hypothetical and generalized behaviors. Future work might build on these by examining actual decisions and behaviors with real consequences. We also note that participants were drawn from modern, Western, highly developed countries with advanced educational systems, so any generalization to very different cultures should therefore be done with caution.

Another limitation pointed out by one of the reviewers, is that the identification of our two process mechanisms, emotion and reputation, was conducted using statistical mediation analysis. This analysis is best described as "observed mediation" rather than "manipulated mediation", in which the latter would normally be the strongest form of causal identification. There is wide agreement on the assertion that random assignment to different levels of the independent variable (i.e. time perspective) enables causal inference of the direct effect on mediators (i.e. emotion and reputation), and the dependent variable (i.e. setting appropriate levels of reward and punishment). However, the indirect pathway from mediators to the dependent variable is merely correlational, since the mediators are not manipulated experimentally. In our view, strong causal inference is not warranted in such an analysis - but the present findings do provide suggestive evidence for the underlying psychological process, when the specific hypothesis is derived from a coherent theoretical framework (Hayes, 2013). In future research on time perspective and moral judgment, it would be helpful to extend the present studies with direct manipulation of the process variables of interest.

A final issue noted by one of the reviewers, was that our procedure first alerted participants to reflect either on the past or the future. It is possible that the effects would have 
been weaker had we simply asked the questions (manipulating only the verb tense of the questions) without the additional sentence. In our view, however, any weakening of the effect would not indicate lesser validity of the hypothesis but simply a weaker test of it insofar as some participants might have assumed the questions referred to all time frames. Hence, we think the initial framing of the study as concerned with either past or future simply produced a stronger and better test of the hypothesis (which was how that instruction was intended).

\section{Concluding Remarks}

A recent article by Uhlmann, Pizarro, and Diermeier (2015) proposed an important shift in the foundation of moral psychology. Whereas most research has focused on how people judge moral actions, Uhlmann et al. proposed that the primary, focal purpose is to judge persons. They suggested that this has a prospective dimension: Ultimately, the pragmatic goal is to know whom one can cooperate with, rely on, and otherwise trust in the future. Judging past actions is a means toward predicting the future, with the focus on individual persons.

The present findings fit well with and even extend that analysis. The orientation toward the future is not limited to judging and predicting the moral character of others but also extends to oneself. If one functional purpose of morality is to promote group cohesion and cooperation in the future, people apparently think that part of that involves raising expectations and standards for their own future behavior as well.

Whether the idealistic ambition and striving for a morally better future are actually justified is beyond the scope of this paper, let alone how to normatively define what a moral future should look like. However, we note that recent evidence has strongly indicated that human society has gradually moved toward less violence and more pro-social behavior throughout history (for review, see Pinker, 2011). The reasons for this so-called moral 
progress are diverse, but on the psychological level we think they might include some of the factors identified in the present work. Improvements in institutions, law, technology, policing, surveillance, and the like have reduced the impunity that allowed people to get away with wicked behavior in the past. In consequence, human life has evolved from being a violent zero-sum game toward a more cooperative positive-sum game, which in itself may serve as a good proxy for moral progress (Pinker, 2011). Along with such cultural changes, emphasizing greater moral consequences for one's actions in the future may indeed be contributing to the slow progress toward a more peaceful and less violent society.

Moralizing the future also has important implications for individual behavior in groups and organizations. A recent literature review by Baumeister, Ainsworth, and Vohs (2016) concluded that groups perform best when people are individually identified and differentiated — and, conversely, the problems and pathologies of groups increase when individuals submerge their identities in the group. Moral responsibility, division of labor among complementary roles, and individual accountability produce positive contributions and enable groups to become more than the sum of their parts. In contrast, free riding, underachievement, and immoral or antisocial tendencies increase when individuality is concealed. In our view, the difference rests partly on moralizing the future. Accountability, for example, means recognizing that one's actions today will have consequences tomorrow. Managers and employees will therefore perform their roles more scrupulously insofar as they connect present actions with heightened moral evaluation of their future selves. Thus, individual accountability and future-orientation may be an especially powerful combination in the psychology of group performance.

Applied implications aside, our primary goal with the series of experiments reported here was to map out the moral mind through a descriptive lens, testing whether morality might be more about the future than the past. At this point, we conclude that the answer is yes. 
As for the psychological "why" question, the effect seems to be driven by the moral mechanisms of emotion and reputational concern, which are stronger in connection with future than past moral choices as well. 


\section{References}

Alicke, M. D. (2000). Culpable control and the psychology of blame. Psychological Bulletin, 126(4), $556-574$.

Balcetis, E., \& Cardenas, A. (2018).Visual experience in self and social judgment: How a biased majority claim a superior minority. Self and Identity, DOI: 10.1080/15298868.2018.1466724.

Bandura, A. (1999). Moral disengagement in the perpetration of inhumanities. Personality and Social Psychology Review, 3(3), 193-209.

Baumeister, R. F. (1990). Suicide as escape from self. Psychological Review, 97(1), 90-113.

Baumeister, R. F. (1991). Escaping the self. New York: Basic Books.

Baumeister, R. F., Ainsworth, S. E., \& Vohs, K. D. (2016). Are groups more or less than the sum of their members? The moderating role of individual identification. Behavioral and Brain Sciences, 39.

Baumeister, R. F., Bratslavsky, E., Finkenauer, C., \& Vohs, K. D. (2001). Bad is stronger than good. Review of General Psychology, 5(4), 323.

Baumeister, R. F., \& Juola Exline, J. (1999). Virtue, personality, and social relations: Self-control as the moral muscle. Journal of Personality, 67(6), 1165-1194.

Baumeister, R. F., Vohs, K. D., DeWall, C. N., \& Zhang, L. (2007). How emotion shapes behavior: Feedback, anticipation, and reflection, rather than direct causation. Personality and Social Psychology Review, 11(2), 167-203.

Baumeister, R. F., Vohs, K. D., \& Oettingen, G. (2016). Pragmatic prospection: How and why people think about the future. Review of General Psychology, 20(1), 3-16.

Caruso, E. M. (2010). When the future feels worse than the past: a temporal inconsistency in moral judgment. Journal of Experimental Psychology: General, 139(4), 610.

Caruso, E. M., Gilbert, D. T., \& Wilson, T. D. (2008). A wrinkle in time: Asymmetric valuation of past and future events. Psychological Science, 19(8), 796-801.

Clore, G. L., \& Huntsinger, J. R. (2007). How emotions inform judgment and regulate thought. Trends in Cognitive Sciences, 11(9), 393-399.

Cohen, J. (1988). Statistical power analysis for the behavioral sciences Lawrence Earlbaum Associates. Hillsdale, NJ, 20-26.

DeWall, C. N., Baumeister, R. F., Chester, D. S., \& Bushman, B. J. (2016). How Often Does Currently Felt Emotion Predict Social Behavior and Judgment? A Meta-Analytic Test of Two Theories. Emotion Review, 8(2), 136-143.

Engelmann, J. M., Herrmann, E., \& Tomasello, M. (2012). Five-year olds, but not chimpanzees, attempt to manage their reputations. PLoS One, 7(10), e48433.

Ferrante, D., Girotto, V., Stragà, M., \& Walsh, C. (2013). Improving the past and the future: A temporal asymmetry in hypothetical thinking. Journal of Experimental Psychology: General, 142(1), 23.

Frijda, N. H. (1986). The emotions: Studies in emotion and social interaction. Edition de La. Cambridge University Press.

Gächter, S., \& Schulz, J. F. (2016). Intrinsic honesty and the prevalence of rule violations across societies. Nature, 531(7595), 496-499. 
Greenwald, A. G. (1980). The totalitarian ego: Fabrication and revision of personal history. American Psychologist, 35(7), 603-618.

Haidt, J. (2001). The emotional dog and its rational tail: A social intuitionist approach to moral judgment. Psychological Review, 108(4), 814-834.

Haidt, J. (2007). The new synthesis in moral psychology. Science, 316(5827), 998-1002.

Hayes, A. F. (2013). Introduction to mediation, moderation, and conditional process analysis: A regression-based approach. Guilford Press.

Helzer, E. G., \& Gilovich, T. (2012). Whatever is willed will be: A temporal asymmetry in. attributions to will. Personality and Social Psychology Bulletin, 38(10), 1235-1246.

Jones, Edward E., Rock, L., Shaver, K. G., Goethals, G. R., \& Ward, L. M. (1968). Pattern of performance and ability attribution: An unexpected primacy effect. Journal of Personality and Social Psychology, 10(4), 317.

Jones, Edward Ellsworth, \& Nisbett, R. E. (1971). The actor and the observer: Divergent perceptions of the causes of behavior. General Learning Press.

Jordan, J. J., Hoffman, M., Bloom, P., \& Rand, D. G. (2016). Third-party punishment as a costly signal of trustworthiness. Nature, 530(7591), 473-476.

Kruger, J., \& Dunning, D. (1999). Unskilled and unaware of it: How difficulties in recognizing one's own incompetence lead to inflated self-assessments. Journal of Personality and Social Psychology, 77(6), 1121-1134.

Leach, C. W., \& Cidam, A. (2015). When is shame linked to constructive approach orientation? A meta-analysis. Journal of Personality and Social Psychology, 109(6), 983.

Mazar, N., Amir, O., \& Ariely, D. (2008). The dishonesty of honest people: A theory of self-concept maintenance. Journal of Marketing Research, 45(6), 633-644.

Morewedge, C. K., \& Buechel, E. C. (2013). Motivated underpinnings of the impact bias in affective forecasts. Emotion, 13(6), 1023-1029.

Norem, J. K., \& Cantor, N. (1986). Defensive pessimism: harnessing anxiety as motivation. Journal of Personality and Social Psychology, 51(6), 1208.

Pagliaro, S., Ellemers, N., Barreto, M., \& Di Cesare, C. (2016). Once dishonest, always dishonest? The impact of perceived pervasiveness of moral evaluations of the self on motivation to restore a moral reputation. Frontiers in Psychology, 7, 586.

Pinker, S. (2011). The better angels of our nature: Why violence has declined. Penguin.

Rand, D. G. \& Nowak, M. A. (2013). Human cooperation. Trends in Cognitive Sciences 17, 413-425

Rothbart, M., \& Snyder, M. (1970). Confidence in the prediction and postdiction of an uncertain outcome. Canadian Journal of Behavioural Science, 2(1), 38.

Rozin, P., \& Royzman, E. B. (2001). Negativity bias, negativity dominance, and contagion. Personality and Social Psychology Review, 5(4), 296-320.

Schwarz, N., \& Clore, G. L. (2003). Mood as information: 20 years later. Psychological Inquiry, 14(34), 296-303.

Seligman, M. E. P., Railton, P., Baumeister, R. F., \& Sripada, C. (2013). Navigating Into the Future or Driven by the Past. Perspectives on Psychological Science, 8(2), 119-141. 
Shepperd, J. A., Klein, W. M., Waters, E. A., \& Weinstein, N. D. (2013). Taking stock of unrealistic optimism. Perspectives on Psychological Science, 8(4), 395-411.

Sjåstad, H. (2019). Short-sighted greed? Focusing on the future promotes reputation-based generosity. Judgment and Decision Making, 14(2), 199-213.

Suddendorf, T. (2013). The gap: The science of what separates us from other animals. Basic Books.

Taylor, S. E., \& Brown, J. D. (1988). Illusion and well-being: A social psychological perspective on mental health. Psychological Bulletin, 103(2), 193-210.

Tomasello, M. (2014). A natural history of human thinking. Harvard University Press.

Uhlmann, E. L., Pizarro, D. A., \& Diermeier, D. (2015). A Person-Centered Approach to Moral Judgment. Perspectives on Psychological Science, 10(1), 72-81.

Vallacher, R. R., \& Wegner, D. M. (1985). A theory of action identification. Hillsdale, NJ: Erlbaum.

Vallacher, Robin R., \& Wegner, D. M. (1987). What do people think they're doing? Action identification and human behavior. Psychological Review, 94(1), 3-15.

Van Bavel, J., Mende-Siedlecki, P., Brady, W. J., \& Reinero, D. A. (2016). Contextual sensitivity in scientific reproducibility. Proceedings of the National Academy of Sciences, 201521897.

Van Boven, L., \& Ashworth, L. (2007). Looking forward, looking back: Anticipation is more evocative than retrospection. Journal of Experimental Psychology: General, 136(2), 289-300.

Vonasch, A. J., Reynolds, T., Winegard, B. M., \& Baumeister, R. F. (2018). Death before dishonor: Incurring costs to protect moral reputation. Social Psychological and Personality Science, 9(5), 604-613.

Watson, D., Clark, L. A., \& Tellegen, A. (1988). Development and validation of brief measures of positive and negative affect: the PANAS scales. Journal of Personality and Social Psychology, 54(6), 1063.

Weinstein, N. D. (1980). Unrealistic optimism about future life events. Journal of Personality and Social Psychology, 39(5), 806.

Wilson, T. D., \& Gilbert, D. T. (2005). Affective Forecasting Knowing What to Want. Current Directions in Psychological Science, 14(3), 131-134.

Ybarra, O., Park, H., Stanik, C., \& Lee, D. S. (2012). Self-judgment and reputation monitoring as a function of the fundamental dimensions, temporal perspective, and culture. European Journal of Social Psychology, 42(2), 200-209.

Zuckerman, M. (1979). Attribution of success and failure revisited, or: The motivational bias is alive and well in attribution theory. Journal of Personality, 47(2), 245-287. 